\title{
Compressible material flow in cylindrical channel with variable cross section
}

\author{
Elena Pryanishnikova ${ }^{1, *}$, Nadezhda Belyaeva ${ }^{1}$, and Alexandr Stolin $^{2}$ \\ ${ }^{1}$ Department of Mathematical Modelling and Cybernetics, Syktyvkar State University named after Pitirim Sorokin, Russia \\ ${ }^{2}$ Institute of Structural Macrokinetics and Material Science of Russian Academy of Science, Russia
}

\begin{abstract}
In the mathematical model of the flow of compressible material the effect of friction and the slip velocity of the material at the side boundary surfaces are considered. The dependence of the slip velocity on the average velocity at the entrance of the channel is built.
\end{abstract}

\section{Introduction}

Mathematical modelling of the compressible frictional flow of material is important theoretical $[1,2,3]$ and practical task $[4,5]$. Corresponding models are applying to describe and to control technological processes of composite material formation. For example, solid-phase plunger extrusion is a process of one-sided compression under press and extrusion. A material is moved from cylindrical chamber in the calibre across conical domain.

\section{Problem statement}

The process of the compressible material flow in a cylindrical channel with variable cross section under the effect of one-sided pressure $\mathbf{P}=\mathbf{P}(\mathbf{t})$ applied to the end surface (fig. 1) is considered. The movement of the material occurs in three areas: cylindrical channel of radius $R_{1}(I)$, transition zone (II), the channel of radius $R_{2}$ (III). A friction force $\mathbf{F}_{\mathbf{f r}}$ between the channel wall and the material counteracts the flow.

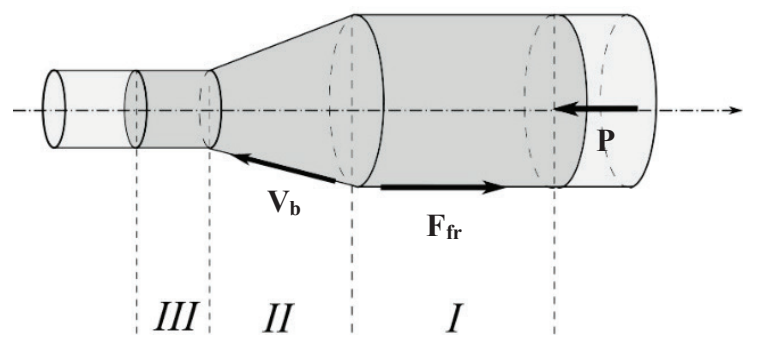

Fig. 1. The model of material flow.

In general, the movement of the material is described by the system consisting the equations for the density determined by the ratio $\rho_{1} \cdot \rho$, where $\rho_{1}$ is density of the incompressible base of the material, $\rho=\rho(r, \varphi, z ; t)$ is relative density, the flow velocity and the stress tensor $\Pi=\left(\sigma_{i j}(r, \varphi, z ; t)\right), \quad i, j=r, \varphi, z$ :

$$
\begin{gathered}
\frac{\partial\left(\rho_{1} \rho\right)}{\partial t}+\operatorname{div}\left(\rho_{1} \rho \mathbf{V}\right)=0, \\
\rho_{1} \rho\left(\mathbf{F}-\frac{d \mathbf{V}}{d t}\right)+\operatorname{div} \Pi=0, \\
\Pi=\left[-p+\left(\xi-\frac{2}{3} \mu\right) \operatorname{div}(\mathbf{V})\right] I+2 \mu \Phi,
\end{gathered}
$$

here (1) is the continuity equation, (2) is the NavierStokes equation [6], $\mathbf{F}$ are mass forces acting on the material. The Newton's law of viscosity (3) is accepted as the differential equation of the state, where $\mu, \xi$ are the variable dynamic and volume viscosity depended on density, $I$ is unit tensor, $\Phi$ is strain rate tensor.

\subsection{Statement of the problem in cylindrical coordinate system}

A cylindrical coordinate system $r, \varphi, z$ is used to describe the problem. The axis of the channel symmetry is assumed as the axis $z$ which positive direction is opposite to the direction of the material movement. At the time $t$ the right-hand boundary of zone $I$ is at the position $z=z_{1}(t)$, the boundary of zone $I I I$ is denoted as $z=z_{3}(t)$. Transition zone $I I$ has a conical shape with a taper angle $\alpha$, its boundaries are constant and denoted as $z=0$ and $z=z_{2}$ . The radius of the cross section of the transition zone is defined by the formula $R(z)=z \tan \alpha+R_{2}$.

Thus the movement of the material occurs in a region with variable boundaries.

\footnotetext{
* Corresponding author: pryanysh@inbox.ru
} 
Due to the symmetry of the channel, the functions do not depend on the angular position and the tangential velocity $v_{\varphi}$, shear stresses $\sigma_{r \varphi}$ and $\sigma_{z \varphi}$ are equal to zero.

In view of the Reynolds number smallness: viscosity of the incompressible base of the material is large, the equation of motion (2) is replaced by the conditions of equilibrium. It is assumed that external forces $\mathbf{F}$ do not exist, pressure $p$ is equal to zero. The motion in zone III is supposed to be unobstructed.

Under these assumptions the equations (1)-(3) in the cylindrical coordinate system with the appropriate initial and boundary conditions in the area $I$ can be written as follows

$$
\begin{gathered}
\frac{\partial \rho}{\partial t}+\frac{1}{r}\left(\frac{\partial}{\partial r}\left(r \rho r_{r}\right)\right)+\frac{\partial}{\partial z}\left(\rho v_{z}\right)=0 \\
\frac{1}{r} \frac{\partial}{\partial r}\left(r \sigma_{r r}\right)+\frac{\partial \sigma_{r z}}{\partial z}-\frac{\sigma_{\varphi \varphi}}{r}=0 \\
\frac{1}{r} \frac{\partial}{\partial r}\left(r \sigma_{r z}\right)+\frac{\partial \sigma_{z z}}{\partial z}=0 \\
\sigma_{r r}=\left(\xi+\frac{4}{3} \mu\right) \frac{\partial v_{r}}{\partial r}+\left(\xi-\frac{2}{3} \mu\right)\left(\frac{v_{r}}{r}+\frac{\partial v_{z}}{\partial z}\right) \\
\sigma_{\varphi \varphi}=\left(\xi+\frac{4}{3} \mu\right) \frac{v_{r}}{r}+\left(\xi-\frac{2}{3} \mu\right)\left(\frac{\partial v_{r}}{\partial r}+\frac{\partial v_{z}}{\partial z}\right), \\
\sigma_{z z}=\left(\xi+\frac{4}{3} \mu\right) \frac{\partial v_{z}}{\partial z}+\left(\xi-\frac{2}{3} \mu\right) \cdot \frac{1}{r} \frac{\partial}{\partial r}\left(r v_{r}\right), \\
\sigma_{r z}=2 \mu\left(\frac{\partial v_{r}}{\partial z}+\frac{\partial v_{z}}{\partial r}\right),
\end{gathered}
$$

initial and boundary conditions

$$
\begin{gathered}
\left.\rho\right|_{t=0}=\rho_{0}, \\
\left.v_{i}\right|_{t=0}=0, i=r, z,\left.\sigma_{i i}\right|_{t=0}=0, i=r, \varphi, z,\left.\sigma_{r r}\right|_{t=0}=0, \\
\left.\sigma_{z z}\right|_{z=z_{1}(t)}=P(t), \\
\left.v_{z}\right|_{r=R_{1}}=v_{b},\left.\quad v_{r}\right|_{r=R_{1}}=0, \\
\left.\sigma_{r z}\right|_{r=R_{1}}=\tau_{f r},\left.\sigma_{r r}\right|_{r=R_{1}}=0,\left.\sigma_{\varphi q}\right|_{r=R_{1}}=0,
\end{gathered}
$$

here $\mu=\mu_{0} \rho^{s}, \quad \xi=\mu \rho /(1-\rho)$. At the initial time the distribution of density, velocity, deformation tensor (12) are set. The relation (13) defines the force in the zone $I$, (14) and (15) define intensity of friction $\tau_{f r}$ and the slip velocity $v_{b}$ at the wall. In particular, in case of sticking $v_{b}=0$.

Let us write the projections of the relations (1)-(3) in region $I I$ :

$$
\begin{gathered}
\frac{\partial \rho}{\partial t}+\frac{1}{r}\left(\frac{\partial}{\partial r}\left(r \rho r_{r}\right)\right)+\frac{\partial}{\partial z}\left(\rho v_{z}\right)=0, \\
\frac{1}{r} \frac{\partial}{\partial r}\left(r \sigma_{r r}\right)+\frac{\partial \sigma_{r z}}{\partial z}-\frac{\sigma_{\varphi \varphi}}{r}=0, \\
\frac{1}{r} \frac{\partial}{\partial r}\left(r \sigma_{r z}\right)+\frac{\partial \sigma_{z z}}{\partial z}=0, \\
\sigma_{r r}=\left(\xi+\frac{4}{3} \mu\right) \frac{\partial v_{r}}{\partial r}+\left(\xi-\frac{2}{3} \mu\right)\left(\frac{v_{r}}{r}+\frac{\partial v_{z}}{\partial z}\right), \\
\sigma_{\varphi \varphi}=\left(\xi+\frac{4}{3} \mu\right) \frac{v_{r}}{r}+\left(\xi-\frac{2}{3} \mu\right)\left(\frac{\partial v_{r}}{\partial r}+\frac{\partial v_{z}}{\partial z}\right), \\
\sigma_{z z}=\left(\xi+\frac{4}{3} \mu\right) \frac{\partial v_{z}}{\partial z}+\left(\xi-\frac{2}{3} \mu\right) \cdot \frac{1}{r} \frac{\partial}{\partial r}\left(r v_{r}\right), \\
\sigma_{r z}=2 \mu\left(\frac{\partial v_{r}}{\partial z}+\frac{\partial v_{z}}{\partial r}\right) .
\end{gathered}
$$

The boundary conditions in the transition zone are written as follows:

$$
\begin{gathered}
\left.v_{z}\right|_{z=0}=-\frac{k_{1}\left|\sigma_{z z}(0, t)\right|^{m}}{\rho_{1} \rho(0, t)},\left.\frac{\partial v_{r}}{\partial z}\right|_{z=0}=0,\left.\frac{\partial \sigma_{r z}}{\partial z}\right|_{z=0}=0, \\
\left.\sigma_{r z}\right|_{r=R(z)}=\tau_{f r} \cos \alpha,\left.\quad \sigma_{r r}\right|_{r=R(z)}=\tau_{f r} \sin \alpha, \\
\left.v_{z}\right|_{r=R(z)}=v_{b} \cos \alpha,\left.\quad v_{r}\right|_{r=R(z)}=v_{b} \sin \alpha .
\end{gathered}
$$

The first relation in (23) sets the velocity at the boundary $z=0$ of zones $I I$ and $I I I$.

\section{Solution of the problem}

To avoid the difficulties associated with the consideration of two-dimensional model (4)-(25) and to analyze the results, the averaging method $[2,7]$, is applied to the system. Average value of function $f(r)$ is defined as

$$
\overline{f(r)}=\frac{2}{R^{2}} \int_{0}^{R} r f(r) d r
$$

Let us write the averaged system in region $I$, using previous designations:

$$
\begin{gathered}
\frac{\partial \rho}{\partial t}+\rho \frac{\partial v_{z}}{\partial z}+v_{z} \frac{\partial \rho}{\partial z}=0, \\
\frac{\partial \sigma_{r z}}{\partial z}-\frac{3}{2 R_{1}} \sigma_{\varphi \varphi}=0, \\
\frac{2}{R_{1}} \tau_{f r}+\frac{\partial \sigma_{z z}}{\partial z}=0,
\end{gathered}
$$




$$
\begin{gathered}
\sigma_{r r}=-\left(\frac{3}{2} \xi+5 \mu\right) \frac{v_{r}}{R_{1}}+\left(\xi-\frac{2}{3} \mu\right) \frac{\partial v_{z}}{\partial z}, \\
\sigma_{\varphi \varphi}=\left(-\frac{3}{2} \xi+4 \mu\right) \frac{v_{r}}{R_{1}}+\left(\xi-\frac{2}{3} \mu\right) \frac{\partial v_{z}}{\partial z}, \\
\sigma_{z z}=\left(\xi+\frac{4}{3} \mu\right) \frac{\partial v_{z}}{\partial z}, \\
\sigma_{r z}=2 \mu\left(\frac{\partial v_{r}}{\partial z}+\frac{3}{R_{1}}\left(v_{b}-v_{z}\right)\right), \\
\left.\rho\right|_{t=0}=\rho_{0},\left.\quad v_{i}\right|_{t=0}=0, i=r, z, \\
\left.\sigma_{i i}\right|_{t=0}=0, i=r, \varphi, z,\left.\quad \sigma_{r z}\right|_{t=0}=0, \\
\left.\sigma_{z z}\right|_{z=z_{1}(t)}=P(t) .
\end{gathered}
$$

System (16)-(25) in the transition zone takes the form:

$$
\begin{gathered}
\frac{\partial \rho}{\partial t}+\frac{2}{R(z)} \rho v_{b} \sin \alpha+\rho \frac{\partial v_{z}}{\partial z}+v_{z} \frac{\partial \rho}{\partial z}=0, \\
\frac{2}{R(z)} \tau_{f r} \sin \alpha+\frac{\partial \sigma_{r z}}{\partial z}-\frac{3}{2 R_{1}} \sigma_{\varphi \varphi}=0 \\
\frac{2}{R(z)} \tau_{f r} \cos \alpha+\frac{\partial \sigma_{z z}}{\partial z}=0, \\
\sigma_{r r}=\left(\xi+\frac{4}{3} \mu\right) \frac{3}{R(z)}\left(v_{b} \sin \alpha-v_{r}\right)+ \\
+\left(\xi-\frac{2}{3} \mu\right)\left(\frac{3 v_{r}}{2 R(z)}+\frac{\partial v_{z}}{\partial z}\right), \\
\left.v_{z}\right|_{z=0}=-\frac{k_{1}\left|\sigma_{z z}(0, t)\right|^{m}}{\rho_{1} \rho(0, t)},\left.\frac{\partial v_{r}}{\partial z}\right|_{z=0}=0,\left.\frac{\partial \sigma_{r z}}{\partial z}\right|_{z=0}=0 . \\
+\left(\xi-\frac{2}{3} \mu\right)\left(\frac{3}{R(z)}\left(v_{b} \sin \alpha-v_{r}\right)+\frac{\partial v_{z}}{\partial z}\right), \\
\sigma_{r z}=2 \mu\left(\frac{\partial v_{r}}{\partial z}+\frac{3}{R(z)}\left(v_{b} \cos \alpha-v_{z}\right)\right) \\
\left(\xi+\frac{4}{3} \mu\right) \frac{\partial v_{z}}{\partial z}+\left(\xi-\frac{2}{3} \mu\right) \frac{2}{R(z)} v_{b} \sin \alpha,
\end{gathered}
$$

\subsection{Determination parameters of the problem}

Assuming that the friction at wall does not depend on the axial coordinate $\partial \tau_{f r} / \partial z=0$, equation (28) can be integrated over $z$ :

$$
\begin{gathered}
\int_{z}^{z_{1}(t)} \frac{2}{R_{1}} \tau_{f r} d s+\int_{z}^{z_{1}(t)} \frac{\partial \sigma_{z z}}{\partial s} d s=0, \\
\frac{2}{R_{1}} \tau_{f r}\left(z_{1}(t)-z\right)+\sigma_{z z}\left(z_{1}(t)\right)-\sigma_{z z}(z)=0 .
\end{gathered}
$$

Using (35) we can express $\sigma_{z z}(z)$ :

$$
\sigma_{z z}(z)=P(t)+\frac{2}{R_{1}} \tau_{f r}\left(z_{1}(t)-z\right)
$$

Integrating the equation (38) we can define an axial component of the stress tensor in zone $I I$

$$
\sigma_{z z}(z)=\sigma_{z z}\left(z_{2}\right)+\frac{2 \tau_{f r} \cos \alpha}{\tan \alpha} \ln \left(\frac{R_{1}}{R(z)}\right) .
$$

We get the gradient of velocity from (31)

$$
\frac{\partial v_{z}}{\partial z}=\frac{\sigma_{z z}}{\xi+\frac{4}{3} \mu}
$$

and integrate

$$
\int_{z_{2}}^{z} \frac{\partial v_{z}}{\partial s} d s=\int_{z_{2}}^{z} \frac{\sigma_{z z}}{\xi+\frac{4}{3} \mu} d s
$$

as result

$$
v_{z}(z)-v_{z}\left(z_{2}\right)=\int_{z_{2}}^{z} \frac{\sigma_{z z}}{\xi+\frac{4}{3} \mu} d s .
$$

To calculate the integral of the right hand part of (44) we use the method of trapezoids. Let us denote it as $J(z)$, then we obtain the recurrent formula for calculating the integral:

$$
\begin{gathered}
J(z)=\int_{z_{2}}^{z} \frac{\sigma_{z z}}{\xi+\frac{4}{3} \mu} d s=J(z-\Delta z)+\int_{z-\Delta z}^{z} \frac{\sigma_{z z}}{\xi+\frac{4}{3} \mu} d s= \\
J(z-\Delta z)+\frac{\Delta z}{2}\left[\frac{\sigma_{z z}(z)}{\xi(z)+\frac{4}{3} \mu(z)}+\frac{\sigma_{z z}(z-\Delta z)}{\xi(z-\Delta z)+\frac{4}{3} \mu(z-\Delta z)}\right], \\
J\left(z_{2}\right)=0 .
\end{gathered}
$$

The equations (26), (27), (29), (30), (32), (36), (37), (39)-(42) are solved numerically by using the method of differences scheme.

\section{Numeric results}

It is assumed that the initial distribution of the material density is given by the function 


$$
\rho_{0}(z)=\rho_{0}+\left(\rho_{m}-\rho_{0}\right) z
$$

the friction intensity is defined as

$$
\tau_{f r}=k_{f r} P(t),
$$

where $k_{f r}$ is the coefficient of the friction. The slip velocity is assumed [5] to be equal to

$$
v_{b}=\beta \tau_{f r}^{c},
$$

here $\beta$ and $c$ are parameters that depend on the material properties. Analysis of the numerical experiment results shows that the process is possible at such values of the coefficient $\beta$ that $\left|v_{b}\right| \leq\left|v_{z}\right|$.

The results of numeric experiments in case of $\beta=0.1 \quad\left(v_{b}=-2.2 \cdot 10^{-5} \mathrm{~m} / \mathrm{s}\right)$ are presented below.

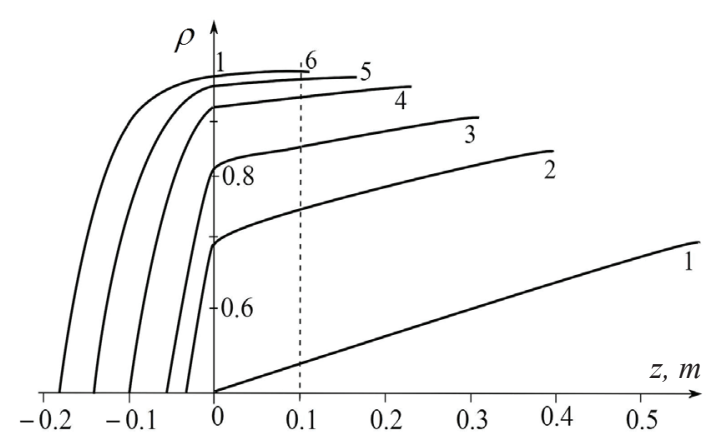

Fig. 2. Distribution of the relative density $\rho=\rho(z ; t)$; full time $503.51 \mathrm{~s}$; full length of billet $0.203 \mathrm{~m} ; \mathrm{t}(\mathrm{s}): 1(0 \mathrm{~s}) ; 2(59 \mathrm{~s})$; 3(114.3 s); 4(224.19 s); 5(279.12 s); 6(443.9 s); 7(498.83 s)

Fig.2 shows the space-time distribution of the relative density. The curves to the right of the axis $\rho$ correspond to the distribution density of the material in zones $I$ and $I I$ (designated by dashed border). It follows from the nature of curves that material is compacted with time, thus the porosity of the material $\Pi=1-\rho$ decreases. This fact, for example, indicates the properties of composite products.

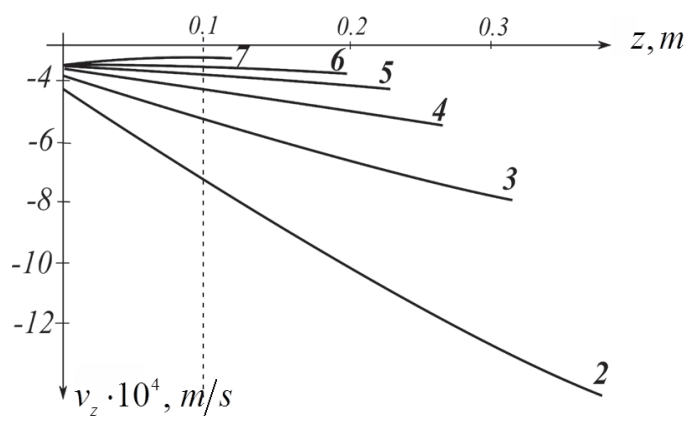

Fig. 3. Distribution of the axial component of velocity $v_{z}=v_{z}(z ; t)$

Velocity, similarly to the stress, decreases from the boundary surface to the hole (fig. 3), the distribution is almost linear. The material becomes more compacted and the velocity values decrease. This effect is accorded with the results obtained in $[1,3,5]$. Due to the changes in the geometric shape and the material density in the region $I I$ the flow of the material is slowed down substantially the velocity is equal to the slip velocity at the walls.

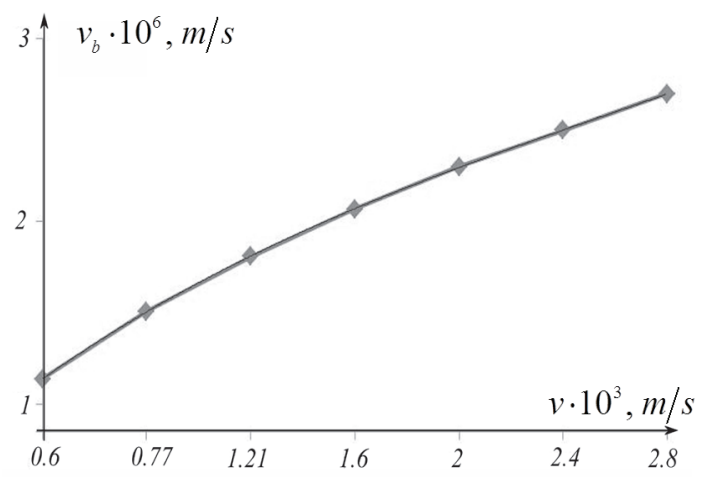

Fig. 4. The dependence of the slip velocity from the average velocity at the inlet of the channel

To determine the dependence of the velocity slip on the walls form the average speed at the entrance to the channel the extensive numerical experiment was conducted, obtained points are represented in fig. 4. This dependence can be approximated by the third degree polynomial

$$
v_{b}=0.0222 v^{3}-0.4345 v^{2}+4.8075 v+7.0143,
$$

which coincides with the results obtained, for example, in [8]. This polynomial can be used to specify the functional dependence of the slip velocity from the velocity on the boundary layer of the material.

\section{Conclusions}

In the research the mathematical model of the composite material flow in cylindrical channel with variable cross section is presented. The form of the boundary conditions is suggested, the numerical experiment was carried out. The dependence estimate of the slip velocity on the average velocity of the material at the entrance is obtained.

\section{References}

1. N.A. Belyaeva, E.A. Pryanishnikova, Vestnik of Syktyvkar University. Ser. 1, 15, 31-44 (2012)

2. E.A. Pryanishnikova, N.A. Belyaeva, Vestnik of Syktyvkar University. Ser. 1, 16, 20-27 (2012)

3. N.K. Evstigneev, A.G. Knyazeva, PNRPU Mechanics Bulletin, 1, 59-71 (2010)

4. A. Perrot, D. Rangeard, Y. Melinge, F. Micaelli, P. Estelle, C. Lanos, Rheologica Acta, 51/8, 743-754 (2012)

5. M. Gupta, ANTEC, 1191-1196 (2011)

6. J. M. McKelvey, Polymer Processing (Khimiya, Moscow, 1965) 
7. S.I. Khudyaev, Threshold phenomena in non-linear equations (Moscow, FIZMATLIT, 2003)

8. I.I. Ivitsky, Technology audit and production reserves, 5/3 (19), 8-11 (2014) 